\title{
Low Complexity Interference Aware Distributed Resource Allocation for Multi-cell OFDMA Cooperative Relay Networks
}

\author{
Nidhal Odeh, Mehran Abolhasan and Farzad Safaei \\ ICT Research Institute, Faculty of Informatics \\ University of Wollongong, Wollongong NSW 2522 Australia \\ \{no999, mehrana, farzad\}@uow.edu.au
}

\begin{abstract}
In this paper we focus on the subcarrier allocation for the uplink OFDMA based cooperative relay networks. Multiple cells were considered, each composed of a single base station (destination), multiple amplify and forward (AF) relay stations and multiple subscriber stations (sources). The effects of inter-cell interference (ICI) have been considered to optimize the subcarrier allocation with low complexity. The optimization problem aims to maximize the sum rate of all sources and at the same time maintain the fairness among them. Full channel state information (CSI) is assumed to be available at the base station. In the proposed algorithm the subcarrier allocation is performed in three steps; firstly the subcarriers are allocated to the Relay Stations (RSs) by which the received ICI on each RS is minimized. Then, the pre-allocated subcarriers are allocated to subscribers to achieve their individual rate requirements. Finally the remaining subcarriers are allocated to subscribers with the best channel condition to maximize the total sum of their data rates. The results show that the proposed algorithm significantly reduces the complexity with almost the same achievable rate of the optimal allocation in a single cell case. In case of multi-cell, the proposed algorithm outperforms the conventional algorithm in terms of total network achievable data rate and overall network complexity.
\end{abstract}

Index Terms-OFDMA, Cooperative Relay, Resource Allocation, Multi-cell, Complexity, Fairness.

\section{INTRODUCTION}

The demand on wireless communications is continuing to grow. This has been mainly due to the increasing popularity of multi-media and internet-type applications. Hence, it is now very important to manage the available resources such as the wireless spectrum more efficiently. Several techniques were proposed to maximize network performance in terms of capacity and quality under limited resources. Among those techniques and proposals, Multiple Input Multiple Output (MIMO) is a technique which uses spatial diversity to transmit in parallel channels simultaneously [1]. One drawback of the aforementioned technique is the system complexity and increased infrastructure cost.

Recently, cooperative communication was proposed as an alternative to MIMO systems, in which the spatial diversity

This work was supported by the Australian Research Council under Grant No. DP0879507: Novel Architecture for Next Generation Wireless Relay Networks. gain can be achieved without the need of multiple antennas at either the transmitter or the receiver. Instead, multiple BSs cooperate to assist each other by relaying each other's signals and thus, MIMO concepts are achieved in a distributed manner [2].

To further minimize the costs, fixed or mobile relay stations were proposed to perform the relaying process. The receiver combines the received signals from the participating relay stations as well as from the source through the direct link using some combination techniques, such as, Maximum Ratio Combining (MRC).

Such RSs will require new resource allocation algorithms and mechanisms, and instead of only considering the direct link between the source and destination nodes, it becomes vital to consider the link quality between the relay and destination as well as between the relay and the source nodes.

Several resource allocation algorithms have been proposed for OFDMA based cooperative relay networks for the purpose of either minimising the transmission power under minimum required data rate constraints [3],[4] or maximizing the data rates under power constraints [5], [6], [7], which is the aim of the this paper.

Most of the previous work considers a single cell scenario, in which ICI is ignored. However, in reality ICI severely degrades system performance and hence, for practical systems it should be considered in the allocation process [8].

In [7], the authors propose a new resource allocation algorithm for OFDMA based cooperative relay networks on the uplink transmission. Fairness constraints were imposed to ensure that the minimum rate is achieved for all sources. However, the ICI was totally ignored and the minimum rate is assumed to be similar for all sources. Furthermore, the algorithm requires an exhaustive search over all combinations of subcarriers and relay stations for each individual source in order to find the best subcarrier-relay pair to be allocated to that source.

The effects of ICI have been considered on the proposed relay selection algorithm in [9]. It is assumed that the source and destination can only communicate through the relay station and the direct link does not exist. Also the interference effects are only assumed to affect the received signals at the relay stations.

In this paper, the ICI is modeled to take into account the effects 
on the relay stations as well as the destination during the first time slot. It is also assumed that the destination receives from the relay stations as well as directly from the source. Moreover, the CSI of each cell is completely known by the serving BS of that cell. This knowledge is obtained through the feedback links between the sources/relay stations and the destination (BS). Under the above assumptions, this paper suggests a new subcarrier allocation algorithm which is able to maximize the total sum rate with low complexity compared to the existing algorithms and able to further increase the total network achievable rate in a multi-cell scenario.

The remainder of this paper is organized as follows: In the next section the adopted system model is introduced. In section III we formulate the optimization problem, and then the proposed subcarrier allocation algorithm is presented and discussed in section IV. The algorithm complexity is discussed in section $\mathrm{V}$ followed by the numerical results in section VI and finally section VII concludes the paper.

\section{System ModeL}

Figure 1, illustrates the adopted system model, in which the proposed network comprises of multiple cells with multiple source nodes $(S)$, multiple relay nodes $(R)$ and a single destination $(D)$. One cell is considered and the rest of the neighboring cells are assumed to be interference sources $(I)$. Moreover, a number of subcarriers $(K)$ are assumed to be available at the destination base station to be assigned to different sources and relay stations. Denote the set of source nodes, relay nodes, interference nodes and subcarriers as $\mathcal{S}=\{1, \ldots, s, \ldots, S\}, \mathcal{R}=\{1, \ldots, r, \ldots, R\}, \mathcal{I}=\{1, \ldots, i, \ldots, I\}$ and $\mathcal{K}=\{1, \ldots, k, \ldots, K\}$ respectively.

Here, it is also assumed that the ICI is generated due to the RSs transmission from the neighbor cells with equal transmit power. Thus, the serving BS has a knowledge about the transmitted power by all other RS's, i.e., $P_{i}$ is known for all $i$ and the transmitted power across each subcarrier $P_{i}^{k}$ is averaged and given by $P_{i}^{k}=P_{i} / K$.

The destination receives the transmitted signal by the source through a direct link between the source and the destination $L_{S D}$ and through the AF relay station links $L_{S R D}$ as shown in Fig. 1, where the straight lines represent the desired signal and the dashed lines represent the interference from neighboring cells.

During the first time slot $\left(T_{s_{1}}\right)$, the source $s$ transmits the signal and both the relays $r, r=1,2, . ., R$ and the destination $D$ receives the transmitted signal, and on the second time slot $T_{s_{2}}$ the RS's forward the amplified copy of the received signal to the destination.

Assuming that the interference only affects the relays and the destination during the first time slot, mathematically, the received signal by the $r^{t h}$ relay station and the destination $(D)$ during $T_{s_{1}}$ over the $k^{t h}$ subcarrier can be respectively written as:

$$
y_{s, r}^{k}=G_{s, r}^{k} \sqrt{P_{s}^{k}} X_{s}+\sum_{i=1}^{I} G_{i, r}^{k} \sqrt{P_{i}^{k}} X_{i}+w_{r}
$$

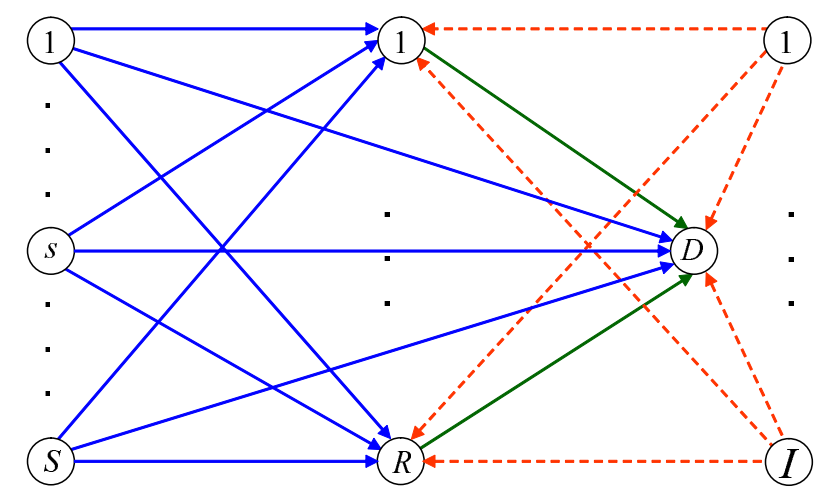

Fig. 1. Multi-Cell Cooperative Relay System Model

$$
y_{s, d}^{k}=G_{s, d}^{k} \sqrt{P_{s}^{k}} X_{s}+\sum_{i=1}^{I} G_{i, d}^{k} \sqrt{P_{i}^{k}} X_{i}+w_{d}
$$

where,

$G_{a, b}^{c}$ Denotes the channel gain between nodes $a$ and $b$ over subcarrier $c$.

$y_{a, b}^{c} \quad$ Denotes the received signal at node $b$ from node $a$ over subcarrier $c$.

$P_{a} \quad$ Denotes the transmitted power from node $a$.

$X_{a} \quad$ Denotes the transmitted signal from node $a$.

$w_{a} \quad$ Denotes the AWGN at node $a$.

during $T_{s_{2}}$, the received signal at the destination from all RSs is given by:

$$
y_{R, d}^{k}=\sum_{r=1}^{R} G_{r, d}^{k} g_{r} y_{s, r}^{k}+w_{d}
$$

$g_{a}$ is the RS amplification factor given by [9]:

$$
g_{r}=\sqrt{\frac{P_{r}^{k}}{P_{s}^{k}\left|G_{s, r}^{k}\right|^{2}+\sum_{i=1}^{I}\left|G_{r, i}^{k}\right|^{2} P_{i}+w_{r s}}}
$$

By substituting (1) in (3), we have

$$
\begin{aligned}
y_{R, d}^{k}= & \sum_{r=1}^{R} G_{r, d}^{k} g_{r} G_{s, r}^{k} \sqrt{P_{s}^{k}} X_{s}+\sum_{r=1}^{R} \sum_{i=1}^{I} G_{r, d}^{k} g_{r} G_{i, r}^{k} \sqrt{P_{i}^{k}} X_{i} \\
& +\sum_{r=1}^{R} G_{r, d}^{k} g_{r} w_{r}+w_{d}
\end{aligned}
$$

The signal to interference and noise ratio (SINR) at the $r^{t h}$ relay station and at the destination from the direct link are respectively

$$
\begin{gathered}
\operatorname{SINR}_{r}^{k}=\frac{\left|G_{s, r}^{k}\right|^{2} P_{s}^{k}}{\sigma_{r}^{2}+\sum_{i=1}^{I}\left|G_{r, i}^{k}\right|^{2} P_{i}^{k}} \\
\operatorname{SINR}_{s, d}^{k}=\frac{\left|G_{s, d}^{k}\right|^{2} P_{s}^{k}}{\sigma_{d}^{2}+\sum_{i=1}^{I}\left|G_{i, d}^{k}\right|^{2} P_{i}^{k}}
\end{gathered}
$$


Considering the $r^{t h}$ RS, the SINR at the destination from the forwarded signal in (5) is given by:

$\operatorname{SIN} R_{r, d}^{k}=\frac{\left|G_{r, d}^{k}\right|^{2} g_{r}\left|G_{s, r}^{k}\right|^{2} P_{s}^{k}}{\sum_{i=1}^{I}\left|G_{r, d}^{k}\right|^{2} g_{r}\left|G_{i, r}^{k}\right|^{2} P_{i}^{k}+\left|G_{r, d}^{k}\right|^{2} g_{r} \sigma_{r}^{2}+\sigma_{d}^{2}}$

By substituting (4) in (8), using the Expressions, $A_{x, y}=$ $\frac{\left|G_{x, y}^{k}\right|^{2} P_{x}^{k}}{\sigma^{2}}, I_{I, d}=\sum_{i=1}^{I} \frac{\left|G_{i, d}^{k}\right|^{2} P_{i}^{k}}{\sigma^{2}}$ and $\sigma_{r}^{2}=\sigma_{d}^{2}=\sigma^{2}$ then, the received SINR at the output of the MRC receiver is written as

$$
\begin{aligned}
& \operatorname{SINR}_{M R C, r}^{k}=\sum_{j=1}^{J} \operatorname{SIN} R_{j, d}^{k} \\
& =\frac{a_{s, r} b_{r, d}}{I_{I, r}\left(b_{r, d}+1\right)+a_{s, r}+b_{r, d}+1}+\frac{c_{s, d}}{1+I_{I, d}}
\end{aligned}
$$

Where $J$ represents the number of the received copies at the destination (through RS's and direct link). It can be seen that equation (9) consists of two parts: the resultant SINR from the relayed links and, the direct link. In [9] the transmission is only assumed through the RSs and thus, the SINR of the direct link is not considered.

Also, it could be noted here that only the ICI is considered and it is assumed that intra-cell interference is zero due to orthogonal subcarriers and perfect synchronization in OFDMA system [10]. However, in case of imperfect synchronization, the multiuser interference (MUI) may occur due to the different carrier frequency offsets between different users. This MUI can be mitigated using some cancellation techniques such as the one proposed in [11].

\section{Problem Formulation}

This section formulates the optimization problem with an objective function to maximize the sum of the source's achievable data rates by imposing fairness (every source receives their minimum rate requirements) and power constraints. The instantaneous rate achieved by the $s^{t h}$ source over the $k^{t h}$ subcarrier with the assistance of the $r^{\text {th }}$ Relay is given by

$$
R_{s, r}^{k}=\frac{1}{2} \log \left(1+S I N R_{M R C, r}^{k}\right)
$$

Assuming all subcarriers are allocated to the source $s$, the total sum rate achieved by the source is given by

$$
R_{s}=\sum_{r=1}^{R} \sum_{k=1}^{K} \rho_{s, r}^{k} R_{s, r}^{k}
$$

and the total achieved rate by all sources and subcarriers is given by

$$
R=\sum_{s=1}^{S} \sum_{r=1}^{R} \sum_{k=1}^{K} \rho_{s, r}^{k} R_{s, r}^{k}
$$

where, $\rho_{s, r}^{k}$ denotes the subcarrier allocation index, given by:

$\rho_{s, r}^{k}= \begin{cases}1 & \text { if subcarrier } k \text { allocated to source } s \text { and relay } r \\ 0 & \text { otherwise }\end{cases}$
The resource allocation optimization problem is then formulated as follows

$$
\max \sum_{s=1}^{S} \sum_{r=1}^{R} \sum_{k=1}^{K} \rho_{s, r}^{k} R_{s, r}^{k}
$$

subject to:

$$
\begin{array}{r}
\rho_{s, r}^{k} \in\{0,1\} \forall k, s, R_{s}^{k} \\
\sum_{s=1}^{S} \sum_{r=1}^{R} \rho_{s, r}^{k}=1 \forall k \\
\sum_{k=1}^{K} P_{s}^{k} \leq p_{s}, \forall s \\
\sum_{k=1}^{K} P_{r}^{k} \leq p_{r}, \forall r \\
P_{s}^{k} \geq 0, \forall k, s \\
P_{r}^{k} \geq 0, \forall k, r \\
\sum_{k=1}^{K} \sum_{r=1}^{R} \rho_{s, r}^{k} R_{s, r}^{k} \geq R_{\min }, \forall s
\end{array}
$$

The constraints (14a) and (14b) indicate that subcarriers are exclusively allocated to one source-relay pair to avoid intracell interference. Constraints (14c)-(14f) are the maximum and the minimum transmission power by each source and relay respectively and constraints $(14 \mathrm{~g})$ are the minimum rate requirements of each source.

It could be seen that the optimization problem in (13) contains both integer (such as, $\rho_{s, r}^{k}$ ) and continuous (such as, $P_{s}^{k}$ and $P_{r}^{k}$ ) decision variables. Thus, the problem is computationally complex. In order to simplify the matter, this paper focuses on the subcarrier allocation rather than power allocation and hence equal power allocation is assumed across subcarriers, i.e., $P_{s}^{k}=P_{r}^{k}=P_{\text {total }} / K, \forall s, r$.

\section{Proposed SubCARrier Allocation}

The optimal allocation can be obtained by joint subcarrier and power allocation for the optimization problem in (13). In our proposed heuristic approach, equal power allocation is assumed across all subcarriers. Thus, the power allocation constraints are ignored and the original problem is simplified to:

$$
\max \sum_{s=1}^{S} \sum_{r=1}^{R} \sum_{k=1}^{K} \rho_{s, r}^{k} R_{s, r}^{k}
$$

subject to:

$$
\begin{array}{r}
\rho_{s, r}^{k} \in\{0,1\} \forall k, s, R_{s}^{k} \\
\sum_{s=1}^{S} \sum_{r=1}^{R} \rho_{s, r}^{k}=1 \forall k
\end{array}
$$

$\sum_{k=1}^{K} \sum_{r=1}^{R} \rho_{s, r}^{k} R_{s, r}^{k} \geq R_{\text {min }_{s}}, \forall s$ 
Taking into account the objective function in (15) and its constraints (16a)-(16c), the proposed subcarrier allocation algorithm is depicted in Algorithm 1. This algorithm consists of four main steps to be performed sequentially. These steps are described in more detail as follows:

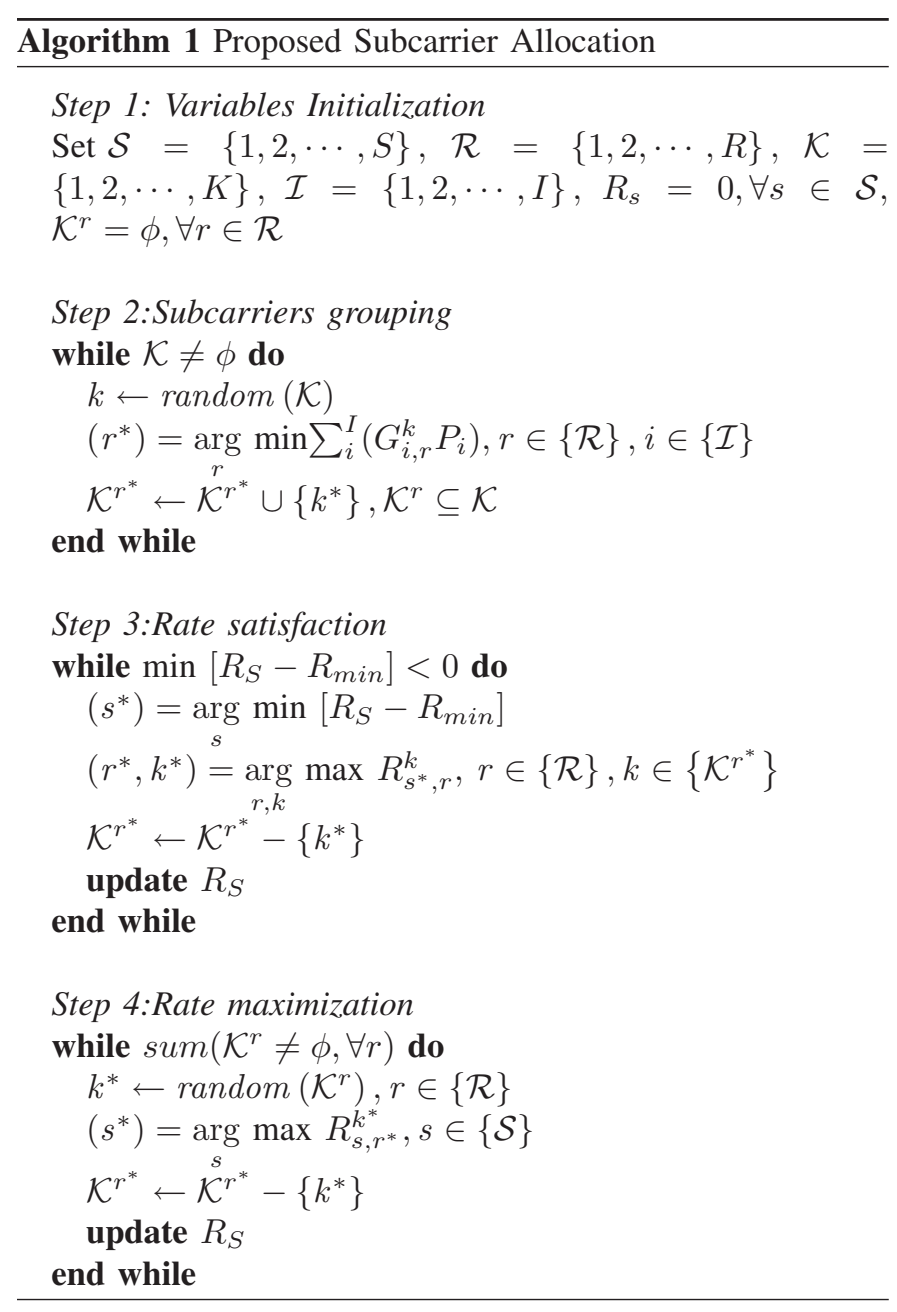

Variable initialization: in this step the sets of variables are initialized.

Subcarrier grouping: This is the main step, in which the subcarriers are grouped into $R$ groups. Each group is assigned to a single RS, by which subcarriers with a high interference on a certain relay station are avoided for that RS. The subcarrier selection for each group (relay) is performed to minimize the ICI on that RS, as the following:

$\left(r^{*}\right)=\arg \min \sum_{i}^{I}\left(G_{i, r}^{(k)} P_{i}\right), r \in\{\mathcal{R}\}, i \in\{\mathcal{I}\}$

Note that by doing this grouping, the BS will reduce the amount of ICI on the neighboring BSs. This is achieved by avoiding subcarriers which have a high negative impact on the neighboring cells.

Moreover, the distributed allocation allows the coordination between neighboring BS without any extra signaling between these BSs, and the global objective of maximizing overall network capacity is obtained in a distributed manner.
The process of assigning the subcarriers to the different groups will continue until every subcarrier joins one of the groups. The output of this step is $R$ groups, each containing a subset of $\mathcal{K}^{r}$ subcarriers. These subsets of subcarriers (groups) are then used in the following step to be allocated to different sources.

Rate satisfaction: in this step, the subcarriers are allocated to different sources to satisfy their individual data rate requirements according to their subscribed service. The fairness between sources is enforced by calculating the difference between the achieved rate and the required rate for each source. The priority of selecting the best subcarrier is given to the one that minimizes this difference, i.e., $\left(s^{*}\right)=\arg \min$ $\left[R_{K}-R_{\min }\right]$. Thus, if the number of subcarriers is not enough to satisfy all sources, this constraint will ensure that all sources will still be able to achieve a rate close to their requirements. This means, sources with high rate requirements will not be allowed to use all the subcarriers at the expense of others.

The source with the highest priority is allowed to select the best subcarrier from any group which maximizes its rate. Note that selecting a certain subcarrier also means selecting the RS to be utilized by that source.

$\left(r^{*}, k^{*}\right)=\underset{r, k}{\arg \max } R_{s^{*}, r}^{k}, r \in\{\mathcal{R}\}, \mathcal{K}^{r} \subseteq \mathcal{K}$

These processes will continue till either the required rates by all sources are achieved or the subcarriers are all allocated. This step is considered as user oriented, because the best subcarriers and relays are assigned to a user based on their requirements.

Rate maximization: In this step we assume that the minimum rate requirements have been achieved by the previous step, thus, this part of the algorithm aims to maximize the total sum rate, and hence this step is considered as subcarrier oriented because the algorithm here tries to find the best user for a certain subcarrier.

It is expected that most of the remaining subcarriers will be allocated to a small subset from the complete set of sources. This is because there should be a group of users having good channel conditions compared to the rest of the users. However, if the sources are mobile users, then this set will continuously change and the users will have a similar probability of having the best channel conditions. Thus, the fairness is also valid here for the long-term for all sources to achieve a maximum data rate. These processes will continue till all of the remaining subcarriers are allocated.

From the last two steps, it can be seen that the algorithm is only focused on allocationg the available subcarriers to different sources rather than to RSs and the search is performed only once for each subcarrier to be assigned to a certain source. This is because the subcarriers are pre-allocated to RSs, and hence sources do not have the choice of selecting a subcarrier from the $r^{t h}$ group and selecting the $j^{t h}$ relay when $j \neq r$, i.e., the RS selection is performed when the source selects the best subcarrier. This significantly reduces the complexity, which will be discussed in the following section. 


\section{Complexity Analysis}

In a conventional approach to allocate to each source the best subcarrier, the algorithm needs to check the maximum achievable rate of that source over all combinations of the available relay stations and subcarriers, hence the complexity associated with each source is equivalent to $\mathcal{O}(R K)$, and for all sources this become $\mathcal{O}(S R K)$. Since $K$ and $S$ are expected to be large compared to the number of relay stations $R$, i.e., $K>S>>R$, then this will significantly increase the system overall complexity.

In the proposed algorithm, the allocation process is divided into two main steps. The first one is to group the subcarriers into $R$ subsets based on minimum ICI with a complexity of $\mathcal{O}(R K)$ which is similar to the conventional algorithm for one source, and then the sources will select a subcarrier from those subsets with a complexity of $\mathcal{O}\left(\sum_{r=1}^{R} K^{r}\right)$, for each source, where $K^{r}$ denotes the number of subcarriers in the subset $\mathcal{K}^{r}$ and $\mathcal{K}^{r} \subseteq \mathcal{K}$. Now considering all sources, and taking into account the grouping complexity, the complexity associated with the overall process becomes $\mathcal{O}\left(R K+\sum_{r=1}^{R} S K^{r}\right)$.

The number of elements in $\mathcal{K}^{r}$ is approximately equal to $\left(\frac{K}{R}\right)$. Thus, although the grouping complexity will increase as the number of relay station increases, this will be compensated during the second step and the complexity is reduced by a factor of $\approx \frac{1}{R}$.

\section{NumericAl Results}

This section presents the numerical results of the proposed subcarrier allocation algorithm. The proposed algorithm has been evaluated in terms of total achievable sum rate of a single cell and multi-cell cases. The results were compared to the conventional algorithm which is a modified version of Jeong algorithm in [7]. The proposed algorithm in [7] assumes equal rate requirements and consider a single cell without ICI. In order to make a fair comparison, the algorithm in [7] was modified by including ICI and assuming different rate requirements for different sources. The sources and relays are distributed randomly within the cell in all cases.

Figure 2 shows the sum rate of the proposed algorithm compared to the conventional algorithm. Here, the sum rate indicates the achievable sum rate of all sources within the cell under consideration. In the case of five sources, it can be seen from the figure that the conventional algorithm outperforms the proposed algorithm especially at high SNR. On the other hand, in the case of a large number of sources (one hundred in this case), we can see that the proposed algorithm behaves exactly the same as the conventional one at any arbitrary SNR value. Thus, the same rate can be achieved with a much simpler algorithm compared to the conventional algorithm.

Moreover, a practical cellular system is very unlikely to have a small number of sources in a cell equipped with four relay stations. Hence the case of five sources is unlikely to happen in a practical cellular system.

To further validate the proposed algorithm, the same simulation was repeated with a different number of sources (users)

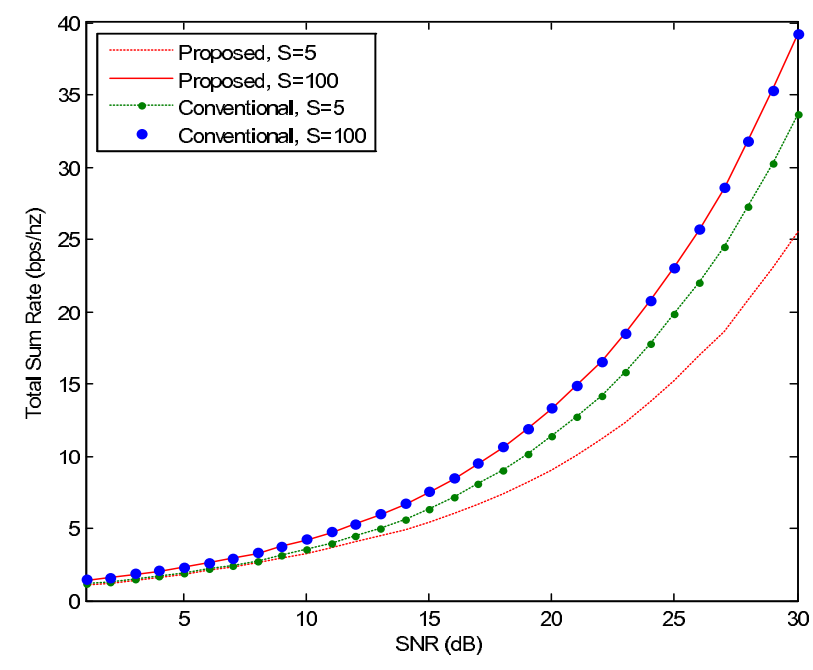

Fig. 2. Total achieved sum rate of the proposed algorithm compared to convention algorithm with $\mathrm{K}=256$ and $\mathrm{R}=6$

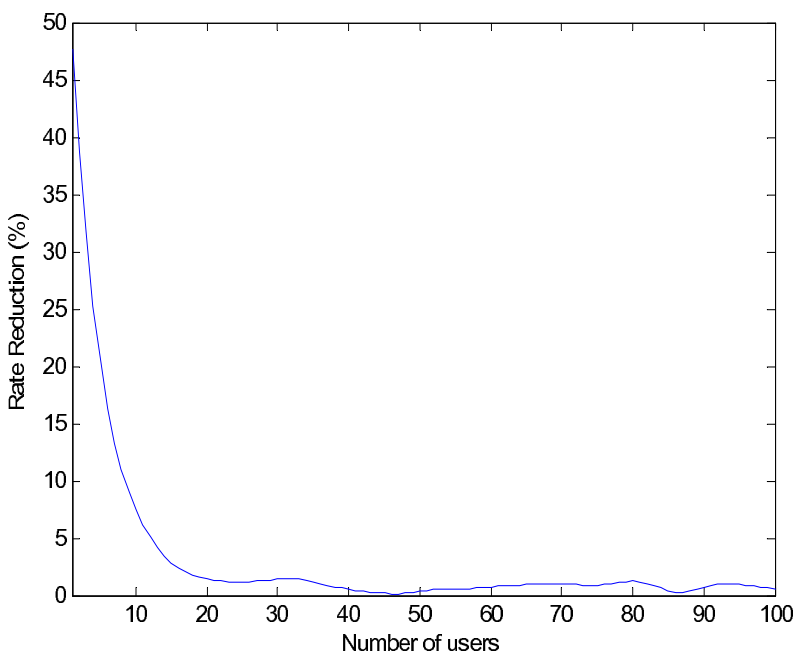

Fig. 3. The Rate Reduction Ratio when adopting the proposed algorithm compared to the conventional one for $\mathrm{K}=256$ and $\mathrm{R}=6$

as shown in Figure 3. Here, the curve presents the difference between the achieved rate of the proposed and the conventional algorithms. It can be seen from the figure that the difference converges to zero as the number of users increases. Moreover, we can see that this difference sharply drops from $45 \%$ to less than 5\% with less than 20 users. Since practical cellular systems accommodate a number of users, in the order of tens or hundreds, it can be said that the proposed algorithm is low in complexity and without any penalty on the sum rate performance.

Taking into account the total network sum rate, Figure 4 shows the total achievable rate of the overall network which consists of two cells, each equipped with four relay stations and 40 randomly distributed sources. The same 256 subcarriers are stored in each BS of both cells to be allocated to the relays and sources.

Although the proposed algorithm achieves a similar perfor- 


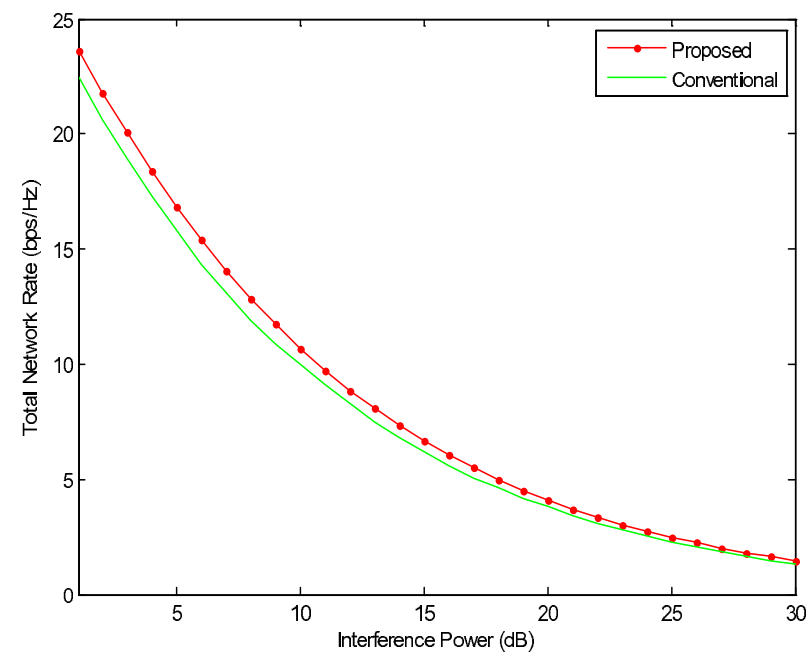

Fig. 4. Total Network Sum Rate, $K=256, S=40,2$ cells each with 4 RSs

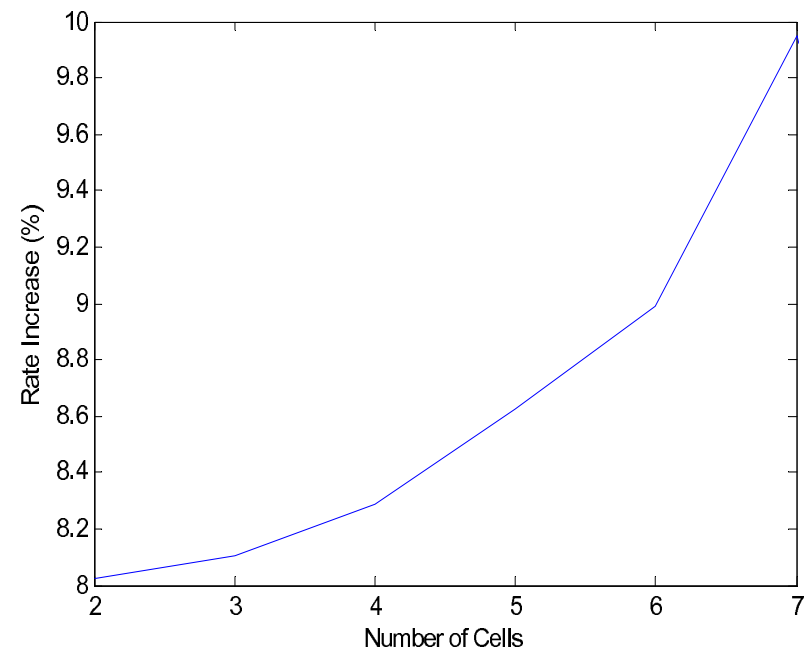

Fig. 5. Total Network Sum Rate with different number of cells, $\mathrm{K}=256$, $\mathrm{S}=40$ and $\mathrm{R}=4$

mance as the conventional one in the single cell case, the key result is that it outperforms the conventional algorithm in the multi-cell scenario. This validates our claims earlier in this paper that in spite of the distributed nature of the proposed algorithm, the ICI is coordinated and minimized in each cell which results in better performance. For the sake of accuracy and reliability, the monte carlo simulation method has been adopted by repeating the same simulation one hundred times using different seed values and the results were averaged out. Furthermore, it can be seen that the gap between the proposed and the conventional algorithm is getting smaller as the interference power increases. This is justified because at high interference power the best subcarrier for each relay still interferes severely with the neighboring one, thus forcing the source to select the best subcarriers among the bad ones. However, the proposed algorithm still outperforms the conventional one. Finally, Figure 5 depicts the achievable rate increment (in percentage) of the proposed algorithm over the conventional one. Different network sizes (i.e., 2 to 7 cells) were considered with the same parameters as in Figure 4 (i.e., $K=256, S=40$ and $\mathrm{R}=4)$. It is clear that the proposed algorithm achieves higher data rate over the conventional algorithm for all network sizes. Furthermore, the figure shows that the increment on the total network rate increases as the number of cells increase. This is because, at high number of cells the coordination becomes more significant to achieve a better rate and hence, the proposed algorithm becomes more efficient.

\section{CONCLUSION}

This paper proposes a new subcarrier allocation algorithm taking into account the ICI in multi-cell environment. The proposed algorithm operates in a distributed manner. The results show that the proposed algorithm's performance is similar to the conventional one in case of single cell. However, the proposed algorithm demonstrates a significant improvement over the conventional one in terms of complexity and required processing time. Moreover, in the multi-cell scenario, the proposed algorithm outperforms the conventional one when measuring the total network achievable rate as well as significantly reducing the overall network complexity.

\section{ACKNOWLEDGMENT}

The author would like to thank Mr. Brett Hagelstein and Mr. Abhinay Mukunthan for their feedback and valuable comments.

\section{REFERENCES}

[1] S. Alamouti, "A simple transmit diversity technique for wireless communications," Selected Areas in Communications, IEEE Journal on, vol. 16 no. 8, pp. 1451-1458, Oct 1998.

[2] A. Sendonaris, E. Erkip, and B. Aazhang, "User cooperation diversity. part i. system description," Communications, IEEE Transactions on, vol. 51, no. 11, pp. 1927-1938, Nov. 2003.

[3] S. Zhou, H. Xiao, and Z. Niu, "Distributed power control for interference-limited cooperative relay networks," in Communications, 2009. ICC '09. IEEE International Conference on, June 2009, pp. 1-6.

[4] J. Yang, D. Gunduz, D. Brown, and E. Erkip, "Resource allocation for cooperative relaying," in Information Sciences and Systems, 2008. CISS 2008. 42nd Annual Conference on, March 2008, pp. 848-853.

[5] M. Awad and X. Shen, "Ofdma based two-hop cooperative relay network resources allocation," in Communications, 2008. ICC '08. IEEE International Conference on, May 2008, pp. 4414-4418.

[6] Z. Shen, X. Wang, and H. Zhang, "Power allocation and subcarrier pairing for ofdm-based af cooperative diversity systems," in Vehicular Technology Conference, 2009. VTC Spring 2009. IEEE 69th, April 2009, pp. 1-5.

[7] H. Jeong, J. H. Lee, and H. Seo, "Resource allocation for uplink multiuser ofdm relay networks with fairness constraints," in Vehicular Technology Conference, 2009. VTC Spring 2009. IEEE 69th, April 2009, pp. 1-5.

[8] Y. Zhu and H. Zheng, "Understanding the impact of interference on collaborative relays," Mobile Computing, IEEE Transactions on, vol. 7, no. 6, pp. 724-736, June 2008.

[9] I. Krikidis, J. Thompson, S. Mclaughlin, and N. Goertz, "Max-min relay selection for legacy amplify-and-forward systems with interference," Wireless Communications, IEEE Transactions on, vol. 8, no. 6, pp. 3016-3027, June 2009.

[10] M. Feng, L. Chen, and X. She, "Uplink adaptive resource allocation mitigating inter-cell interference fluctuation for future cellular systems," in Communications, 2007. ICC '07. IEEE International Conference on, June 2007, pp. 5519-5524.

[11] S. Manohar, D. Sreedhar, V. Tikiya, and A. Chockalingam, "Cancellation of multiuser interference due to carrier frequency offsets in uplink ofdma," Wireless Communications, IEEE Transactions on, vol. 6, no. 7, pp. 2560-2571, July 2007. 